\title{
Center Aspirated Ejector Suction Performance of Numerical Simulation

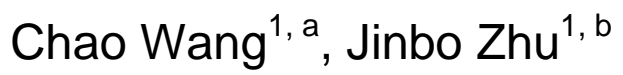

\author{
${ }^{1}$ Materials Science and Engineering, Anhui University of Science \& Technology, \\ Huainan, 232001, China
} \\ awangchao1987wc@163.com, boppooppo5@163.com
}

\begin{abstract}
Keywords: CFD simulation technique, Compound flotation machine, Injector, Suction
\end{abstract}
\begin{abstract}
Article briefly describes the application of CFD simulation technique and experimental validation in the development, improvement and optimization of flotation machine, meanwhile the paper designs an injector used for compound flotation machine according to working principle. The conical injector with shrinking angle $\alpha=13^{\circ}$ and the straight conical injector with shrinking angle $\alpha=13^{\circ}$ and length-diameter ratio $1 / \mathrm{d}=3$ are simulated by fluid dynamics software fluent. The paper analyzes emphatically the suction property of the two kinds of injector under the condition of different distance from the suction pipe to outlet. The results show that the suction property of the straight conical injector is superior to the conical injector. The suction property is the best for straight cone injector when distance between suction pipe and outlet within 14 22 mm.
\end{abstract}

\section{Introduction}

Jet flotation machine is a kind of inflatable flotation machine, whose core component is aeration stirring device. It is made up of the nozzle and throat, the umbrella-type distributor, spray chamber and the suction pipe. In the aggregate, the aeration stirring device as part of the jet device, namely kinds of two phases flow injector. Generally, two phase flow injector consist of nozzle, gas entrainment chamber, mixing tube and diffusion tube. Fluid from the high-speed jet nozzle is caused by sudden narrowing in throat diameter, fluid velocity, pressure decreases, so that the ejector fluid (especially gas) is pumped into the suction chamber, after two kinds of fluid in pipe mix. Jet flotation machine is the application of the principle of injector in mineral processing machinery, with the high-speed jet ejector pumping air and the distributor by the throat, umbrella oblique to the false bottom gas stirring.

The CFD software has been used in the study of two-phase flow injector work increasingly. The present studies focus on the flotation tank structure design, bubble generation and process control, optimization of structural parameters of injector and the analysis of the internal flow field characteristics. For enhancing the separation efficiency of flotation methods by improve fluid dynamics. Yan Xiaokang and Liu Jiongtian [1] used Computational Fluid Dynamics simulation to model the fluid environment of a cyclonic-static micro bubble flotation column. The complicated flow inside the generator resulted in high $R \& D$ costs and difficulty in testing. LI Lin and LIU Jiongtian [2] used the CFD software, Fluent to simulate the gas-liquid two-phase flow inside a self-absorbing micro bubble generator. The effect of area ratio, a key structural parameter, was studied in detail. Critical flow-field parameters including velocity, turbulent kinetic energy, minimum static pressure and gas holdup were obtained. The simulation results demonstrate that the optimum area ratio is 3. Deng Xiaowei, Liu Jiongtian [3] studied the flow field velocity distribution in both cross section and longitudinal section within cyclonic zone for different circulating volumes. The cross sectional vortex was also analyzed. Li Yan [4] used ANSYS simulation technology and finite volume method and the standard $\mathrm{k}$ - epsilon zero equation turbulent model in the air - water mixed, fully considered the affect of nozzle and mixing chamber and the divergent tube on jet performance, the jet device had been simulated for the full flow field, and the simulation results had been validated by the field experiment. Yao Yun [5] simulates the fluid mechanics and mixing characteristics in gas liquid two phase under different feeding way (gas imported centerline and 
liquid imported centerline offset) inside the ejector by using fluent. Song Likai [6] used the computational fluid dynamics software Fluent to simulate the steam ejector, research the nozzle throat diameter and uniform section mixing chamber diameter and length, and other geometrical parameters, which affect the performance of the ejector. Shi Lin [7] studied on the flow field within the gas-solid injector and the behavior of particles by numerical simulation research.

On this basis, designed a new type center breathe ejector for flotation device, simulated the cone and cone shape ejector by using the fluent software and analyzed emphatically the effects of suction quantity of ejector under the condition of different distance from the export.

\section{Experiment Approach}

Yang Guolai [8-9], etc simulated the conical nozzle and the cone shape nozzle under different structural parameters in the research of high pressure water jet. For the cone convergent nozzle, axial velocity would achieve the best when the nozzle contraction angle set at $12 \sim 16^{\circ}$; For the cone shape of the nozzle, the axial velocity would achieve the best when the nozzle contraction angle set at $12 \sim 15^{\circ}$. The best of the nozzle length to diameter ratio set at 2 4. Liu Jun [10] researched the efficiency of hitting the target in 5 kinds of conical nozzle. The results showed that the cone shape of the nozzle has the largest effective distance and the highest efficiency. The basic characteristics of the nozzle set at $13^{\circ}$ and the best of nozzle length to diameter ratio set at 2 4.

Considering the above characteristics of nozzle, the contraction angle of the jet nozzle set at $13^{\circ}$ and the length to diameter ratio set at 3 in this paper. The structural parameters of the conical nozzle as shown in Fig. 1 (a), the structural parameters of the cone shape nozzle as shown in Fig. 1 (b). The high pressure water jet from feeding tube into the nozzle, because of high speed jet, the negative pressure is generated in the end of the nozzle and the air is inhaled through the suction port, the gas and liquid mixed and ejected from export at a certain speed.

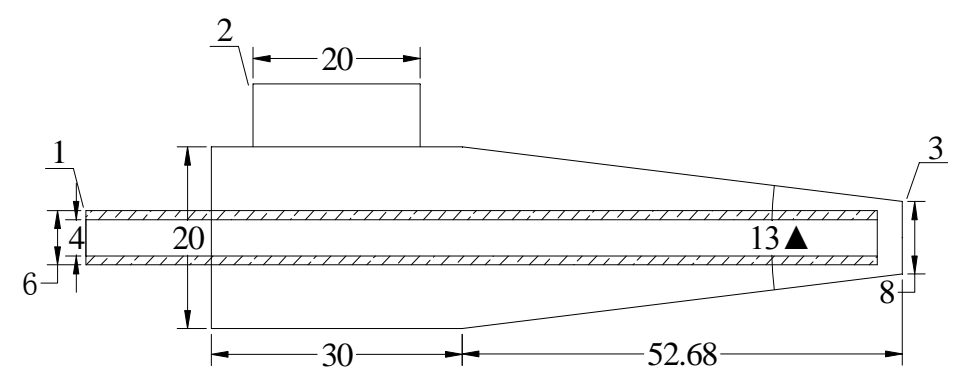

(a) Structure diagram of conical ejector.

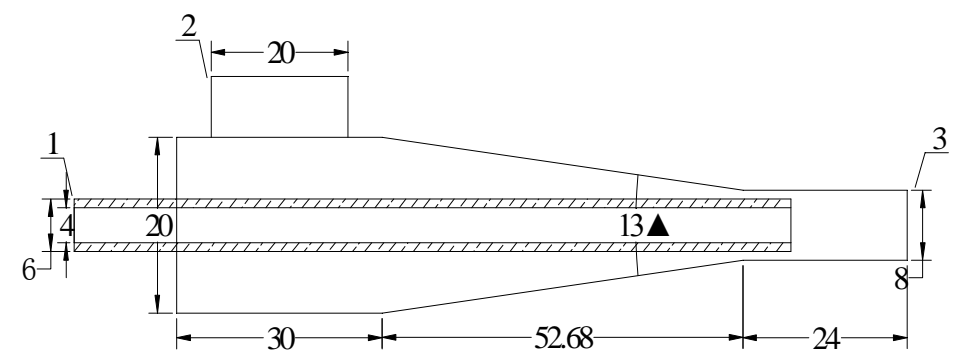

(b) Structure diagram of straight cone shaped ejector.

Fig. 1 Structure diagram of new spray inhalation device.

1 intake pipe; 2 feed tube; 3 exports

\section{Simulation Conditions}

Simulate the gas-liquid flow in ejector three-dimensional by using the fluent software. In the simulation, set the air density as gas, and the water as liquid. Elected the Euler model for multiphase flow model, while normal k-s model for turbulence model. Operating pressure is $0 \mathrm{~Pa}$, which 
represents absolute pressure, without gravity conditions. Boundary conditions: water inlet pressure for import, set the pressure as $150000 \mathrm{~Pa}$, gas inlet pressure for import, set table press $0 \mathrm{~Pa}$. Exports and atmosphere are interlinked; table press is $0 \mathrm{~Pa}$. Considering the wall thickness of ejector suction in the process of simulation the influence of the internal flow field, the wall thickness is set to 1 $\mathrm{mm}$.

Table 1. Suction pipe space from exit.

\begin{tabular}{lll}
\hline \multicolumn{2}{l}{ Ejector form } & The suction space from exports $(\mathrm{mm})$ \\
\hline 1 & The conical ejector & $2,3,4,5,6,8$ \\
2 & The cone shape ejector & $4,9,14,16,18,20,22,24,26,28$ \\
\hline
\end{tabular}

\section{Simulation Results}

(1) Effects of different distance from intake pipe to the outlet of nozzle on the cone ejector

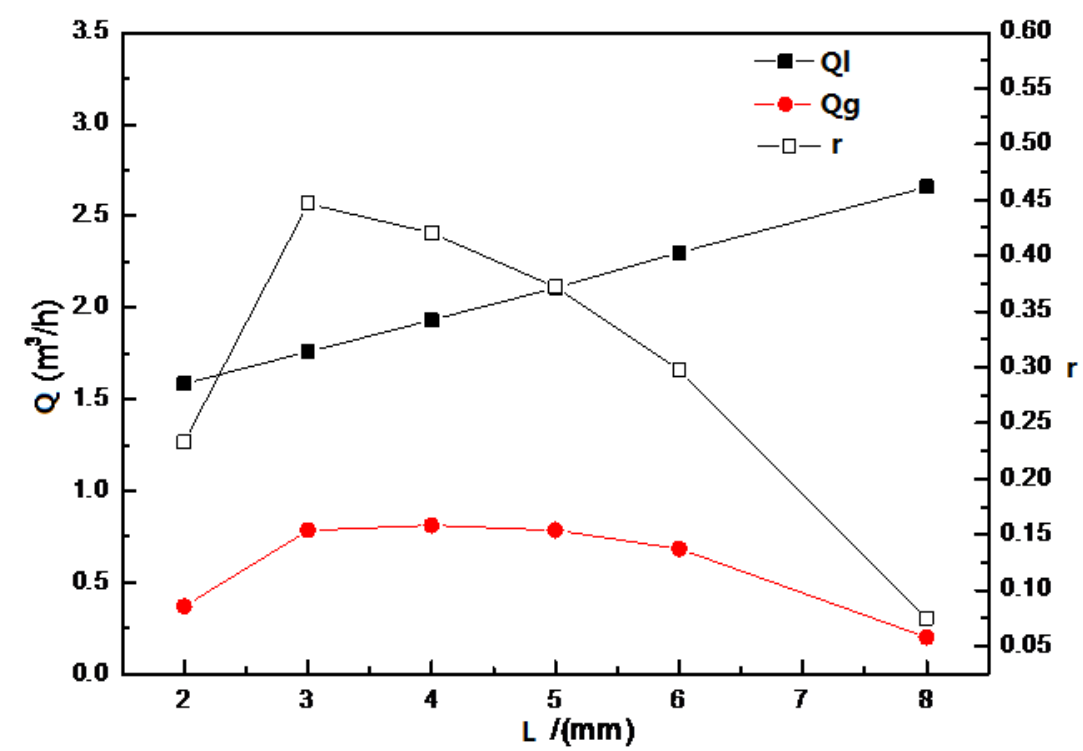

Fig. 2 Diagram of gas flow, liquid flow and gas-liquid ratio of conical nozzle.

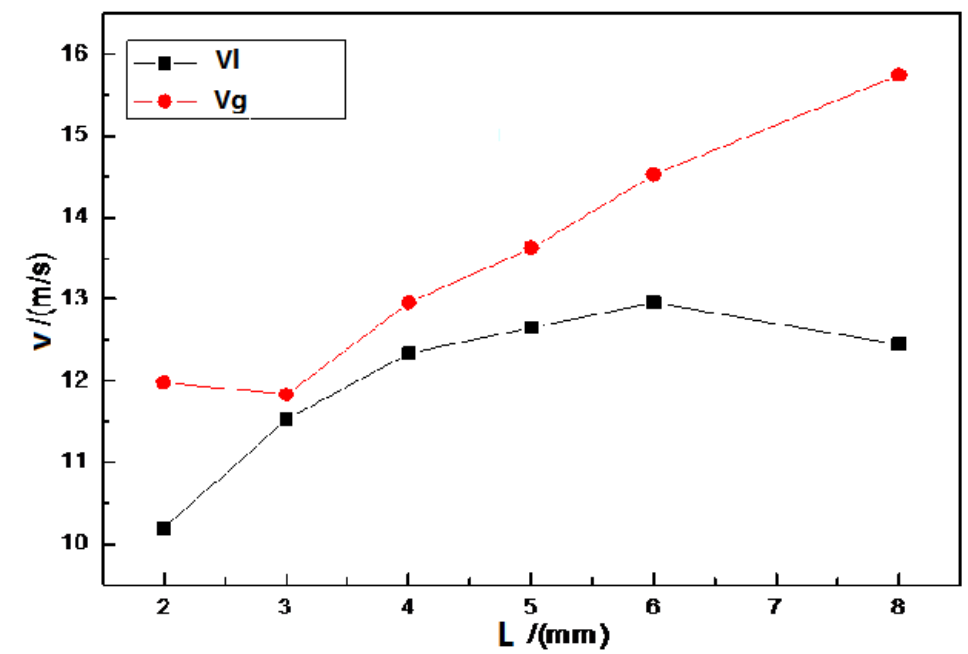

Fig. 3 Diagram of conical spray export gas velocity and liquid velocity.

Suction tube distance away from exports of conical spray as shown in Fig. 2 and Fig. 3. As showed in Fig. 2, with the increasing of the distance of the suction pipe from the export, the liquid flow increases in linear relation. The gas flow shows a parabola relationship and reaches maximum $3 \mathrm{~mm}$ away from the outlet, while the liquid flow is small. So the ratio of gas and liquid reaches the largest, about 0.45. Fig. 3 shows that the speed of liquid and gas are in the form of a parabolic curve. The liquid rate can reach the top speed, and the gas rate can reach the lowest speed. The two speed 
curve of gas and liquid intersect in the bottom of the gas velocity, when the distance of the suction tube away from exports is $3 \mathrm{~mm}$. In the other word, in $3 \mathrm{~mm}$ the speed of gas is close to the speed of liquid place gas and the mixing effect of gas and liquid is best. Combined with Fig. 2 you can see, the ratio of gas and liquid is 0.45 at this time.

(2) Effects of different intake pipe from the outlet for the cone straight ejector

Suction tube distance from export influence on straight cone shaped ejector as shown in Fig. 4 and Fig. 5. As showed in Fig. 2, along with the increase of the suction tube from exports, liquid flow rate increased gradually. From the outlet of the $24 \mathrm{~mm}$, just in the junction of the conic section and straight segment, the liquid flowed growth. Gas also flowed along with the increase of the suction space. However, in contrast to the liquid flow rate, the gas flow began to slow and presented a down trend the outlet of the $24 \mathrm{~mm}$. The junction of conic section and straight section is a turning point. It can be found from the gas and liquid ratio curve that the gas liquid ratio curve appears gradually increased to relatively stable and decreases sharply. For example, the gas liquid ratio increased gradually in the export of $0 \sim 14 \mathrm{~mm}$ range. But in the 14 22 $\mathrm{mm}$ range, gas liquid ratio change is small, stable at around 1.15. After more than $24 \mathrm{~mm}$, the gas liquid ratio decreases obviously.

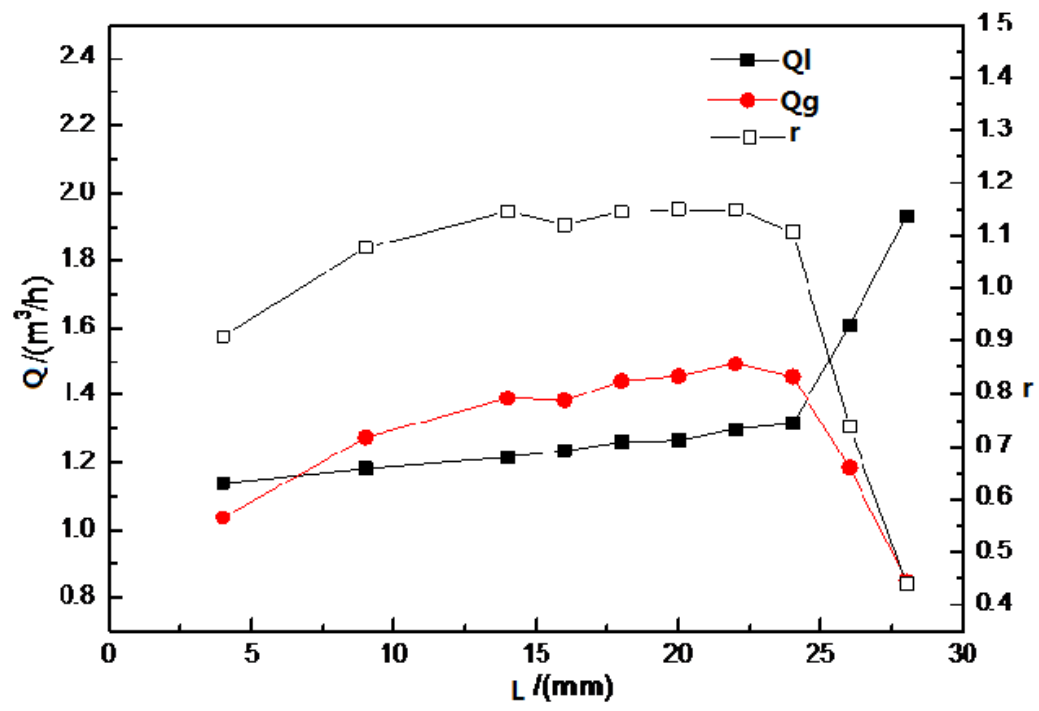

Fig. 4 Diagram of gas flow, liquid flow and gas-liquid ratio of straight cone shaped ejector.

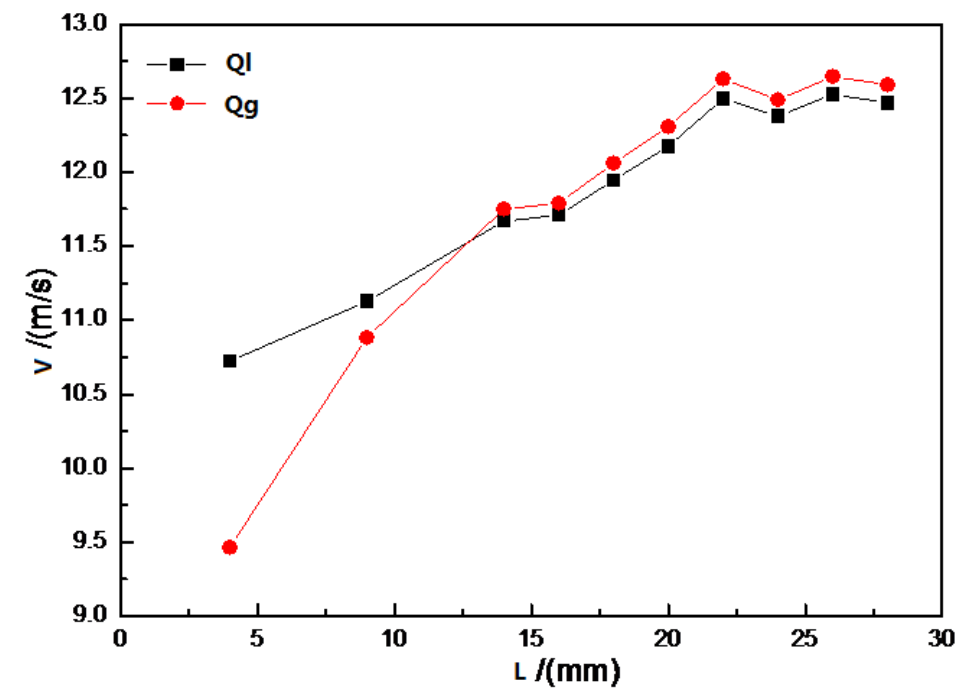

Fig. 5 Diagram of straight cone shaped ejector export gas velocity and liquid velocity.

As shown in the Fig. 5, gas and liquid velocity curve generally showed a gradual increasing trend. From $14 \mathrm{~mm}$, the speed of the gas phase and liquid phase almost same, which means the gas-liquid mixing effect is good. In the $22 \mathrm{~mm}$, it got the maximum, and then a small amount of 
decline.

(3) Velocity vector diagram of the two ejector gas-liquid mixing

Compared of Fig. 2 and Fig. 4, the highest gas-liquid ratio of conical spray can reach 0.45 . The cone shape ejector gas-liquid ratio is around 1 generally, and the highest can reach 1.15 . The causes of this phenomenon can be explained from the diagram of the velocity vector on two ejectors gas-liquid mixing.

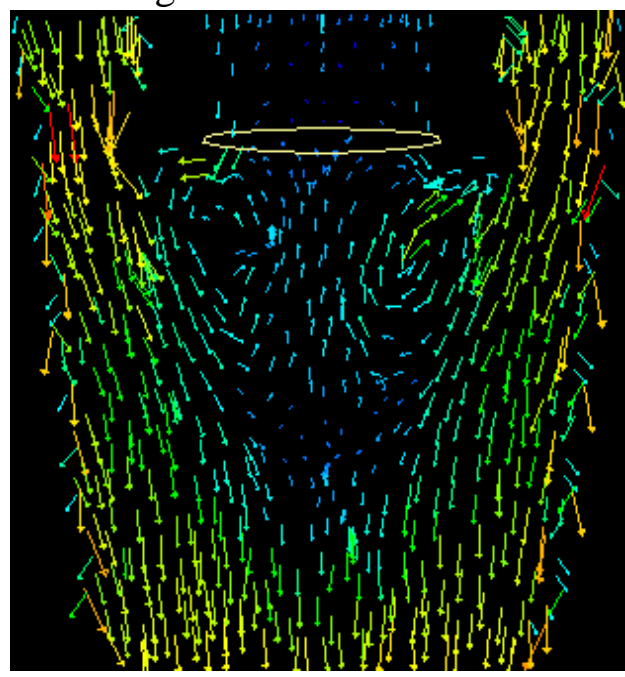

(a) Conical spray

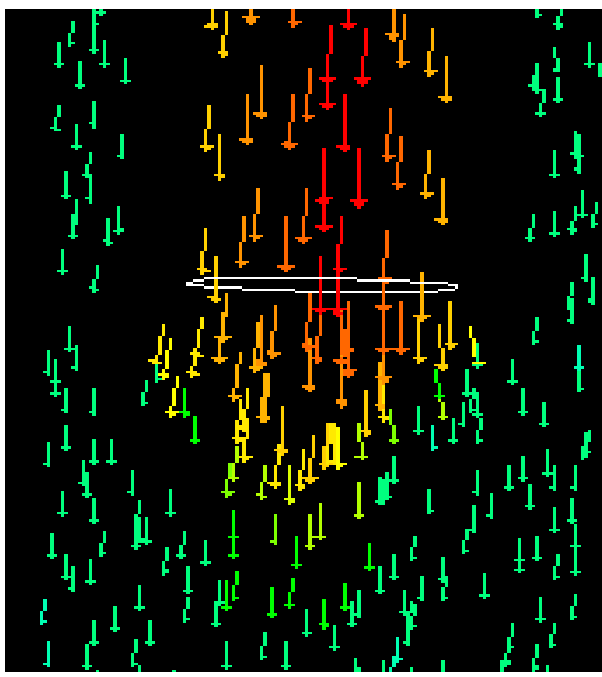

(b) Straight cone shaped ejector

Fig. 6 Diagram of mixed phase velocity vector in suction nozzle exit.

As Fig. 6 (a) shows, the gas in the air suction pipe outlet formed the reverse eddy current, which is not conducive to the suction effect. This is because the conical intake pipe injector is conical converging section; in where the speed feed direction is obliquely along the inner wall of the ejector. It is easy to form the gas vortex in reverse at the outlet of the suction duct at oblique force, impede the process of breathing. This also explained why when the cone straight ejectors suction tube from the outlet more than $24 \mathrm{~mm}$ (cone convergent section and straight section of the junction), the intake was significantly decreased.

Fig. 6 (b) shows that it is a good draw gas when the suction tube in a straight cone shaped ejector straight segment, the speed of liquid vertically downward direction where not produce eddy current in the suction exports.

\section{Conclusion}

(1) Due to the suction nozzle in spray inhalation device inside, it is necessary to consider the influence of suction pipe wall on the internal flow field. And gas-liquid mixing concentrated near the injector outlet, so the appropriate encryption near grid will help to get accurate results.

(2) The cone shape breathe in overall performance is better than that of conical spray injector.

(3) For straight cone shaped ejector whose export is $8 \mathrm{~mm}$ diameter breathe in the optimal performance, in which $\alpha=13^{\circ}, 1 / \mathrm{d}=3$ and suction tube from export within 14 22 $\mathrm{mm}$ spacing, breathe in the optimal performance.

\section{Acknowledgement}

In this paper, the research was sponsored by the NSFC (Natural Science Foundation of China (Project No. 51374015)).

\section{References}

[1] X. K. Yan, J. T. Liu. A single-phase turbulent flow numerical simulation of a cyclonic-static micro bubble flotation column. Int. J. Mining Sci. Technol. 22(1) (2012) 95-100. 
[2] L. Li, J. T. Liu. Numerical simulation of a self-absorbing microbubble generator for a cyclonic-static microbubble flotation column. Mining Sci. Technol. 20(1) (2010) 88-92.

[3] X. W. Deng, J. T. Liu. Velocity distribution of the flow field in the cyclonic zone of cyclone-static micro-bubble flotation column. Int. J. Mining Sci. Technol. 23 (1) (2013) 89-94.

[4] Y. Li, Z. X. Wei, Air - water mixed ANSYS simulation and throat optimization of jet. J. Changchun U. Sci. Technol. (Natural Sciences). 22(6) (2003) 110-112.

[5] Y. Yao, S. Q. Zheng. The fluid flow and mixing characteristics of the gas-liquid ejector with different feeding ways. J. Qingdao U. Sci. Technol. (Natural Science) 5 (2008) 426-431, (in China).

[6] L. K. Song, S. C. Liu. Analysis of the influence of geometric parameters on the ejector performance. Refrigeration 41 (2014) 56-59, (in China).

[7] L. Shi, X. C. Lin. Three-dimensional numerical simulation of gas-solid injector based on Fluent. Coal Preparation Technol. 4 (2011) 19-23, (in China).

[8] G. L. Yang, X. H. Li, W. H. Zhou. Conical nozzle structure parameters on the influence of the jet flow field. J. Hydr. Pneum. 5 (2009) 62-64, (in China).

[9] G. L. Yang, L. N. Chen, X. H. Li. The Influence of Conical Nozzle Geometric Parameters on the Property of Zfflux Flow Field. J. Hydr. Pneum. 11 (2009) 6-8, (in China).

[10]J. Liu, Z. Qin. Nozzle Design Principle and Basis in High-Pressure Water Jet Cleaning. Pipeline Technique and Equipment. 5 (2007) 43-44, (in China). 